\title{
A Study of the Protective Effect of Oral Rehydration Solution on Cisplatin-induced Nephrotoxicity in Rats
}

\author{
Junya Sato ${ }^{* 1,2}$, Katsuo Takahashi, ${ }^{1,2}$, Kazuo Hino ${ }^{3}$ and Kenzo Kudo ${ }^{1,2}$ \\ Department of Pharmacy, Iwate Medical University Hospital ${ }^{1}$, \\ Department of Clinical Pharmaceutics, School of Pharmacy, Iwate Medical University ${ }^{2}$, \\ Nutrition Research Laboratory, Otsuka Pharmaceutical Factory, Inc ${ }^{3}$ \\ $\left[\begin{array}{l}\text { Received October 21, } 2013 \\ \text { Accepted December 13, } 2013\end{array}\right]$
}

Chemotherapy regimens using cisplatin (CDDP) have rarely been applied to outpatients because they require vigorous hydration to reduce CDDP-induced nephrotoxicity. In this study, we investigated the protective effect of oral rehydration solution (ORS) on CDDP-induced nephrotoxicity in rats, which could be applicable to outpatients for oral hydration.

Two mg/kg CDDP was intraperitoneally injected into male F344 rats $(\mathrm{n}=8)$ once a week for 7 weeks. A total of 60 $\mathrm{mL} / \mathrm{kg} /$ day water, ORS, or modified ORS (MOS: chloride-free ORS) was orally administered for hydration. Changes in renal functions were evaluated by plasma creatinine, creatinine clearance, and histopathological evaluation of the kidney. Urinary excretion of platinum and chloride was also measured.

Evaluations of body weight (205 \pm 13 vs $238 \pm 7 \mathrm{~g}, P=0.002)$ (Mean $\pm \mathrm{SD}$, water vs ORS), serum creatinine $(0.69 \pm 0.19$ vs $0.41 \pm 0.05 \mathrm{mg} / \mathrm{dL}, P=0.023)$, creatinine clearance $(0.48 \pm 0.22$ vs $0.74 \pm 0.23 \mathrm{~mL} / \mathrm{min}, P=0.044)$, and histopathological evaluation at the 7 th week revealed that nephrotoxicity was prevented more effectively by oral hydration using ORS than using water. MOS did not show any protective effects. Urinary platinum excretion after CDDP administration was similar between water and ORS. Urinary chloride concentration was higher after ORS administration.

ORS reduced CDDP-induced nephrotoxicity in rats, probably through providing chloride ions to prevent CDDP activation in renal tubules and to promote CDDP excretion. As the clinical application of ORS in cisplatin regimens may contribute to release from infusion, the shortening of the bed residence time and patient's quality of life improvement, safety and usefulness of ORS during cisplatin chemotherapy need to be evaluated.

Key words — oral rehydration, cisplatin, outpatients, chemotherapy, chloride

\section{Introduction}

Cisplatin (CDDP) remains an important component of chemotherapy regimens for a wide variety of solid tumors including ovarian, gastric, testicular, head-neck, and lung cancer. ${ }^{1)}$ Owing to its nephrotoxicity, which is the major dose-limiting effect, chemotherapies using cisplatin require vigorous hydration to reduce cisplatin-induced nephrotoxicity.

Recently, it has been reported that cisplatin administration was feasible in outpatient manage- ment with a short hydration regimen without a high risk of nephrotoxicity. ${ }^{2,3)}$ There are also some reports of oral hydration as a supplement after short intravenous hydration or a substitute of infusion, but the safety and usefulness of oral hydration have not been fully established. ${ }^{4,5)}$

Outpatient chemotherapy with cisplatin is also attempted in Japan in order to improve the quality of life of patients and to cope with the significant load on the available inpatient facilities, but the majority of departments adopt inpatient chemotherapy with a relatively large volume of intrave-

* 19-1 Uchimaru, Morioka, Iwate 020-8505, Japan 
nous hydration to ensure safe administration.

Oral rehydration solution (ORS), which was originally developed and spread by the World Health Organization in developing countries to treat acute diarrheal illness, is now utilized to treat gastroenteritis in developed countries. ${ }^{7,8)}$ The mechanism essential to the efficacy of ORS is the coupled transport of sodium and glucose molecules at the intestinal brush border. Water passively follows the osmotic gradient generated by the transcellular transport of electrolytes and nutrients. The composition of ORS is characterized by relatively high sodium and relatively low sugar (glucose) concentrations compared with those of commercially available sports drinks.

Besides gastroenteritis, ${ }^{9)}$ ORS was shown to be safe and effective as a substitute of infusion during the perioperative period. ${ }^{10,11)}$ Considering its ability to replenish water and electrolytes, which is comparable to that of intravenous solutions, ORS seems to be an appropriate beverage for oral hydration in outpatient chemotherapy. In our previous study, oral hydration with ORS but not with water prevented CDDP-induced nephrotoxicity in rats. ${ }^{12}$

Although the prevention of CDDP-induced nephrotoxicity by vigorous hydration with saline is thought to be the result of promotion of the urinary excretion of CDDP, providing chloride ions to reduce the formation of toxic agents and the induction of osmotic stress response in tubular cells, ${ }^{13)}$ it is unclear whether these mechanisms were involved in the protective effect of oral hydration with ORS against CDDP-induced nephrotoxicity in our experiment.

In the present study, we examined the mechanism of the protective effect of oral hydration with ORS in CDDP-induced renal injury rats, from the perspectives of urinary CDDP excretion and the supplementation of chloride ions.

\section{Materials and Methods}

\section{Administration of CDDP and hydra- tion schedule}

Forty male Fischer-344/DuCrlCrlj rats (Charles River Laboratories Japan, Inc, Yokohama, Japan), 7 weeks old, weighing 125-155 g, were used. They were kept in an animal room controlled at $23 \pm 3{ }^{\circ} \mathrm{C}$ with a 12 -hr light/dark cycle. All animals were given a standard commercial laboratory diet for rats (CRF-1; Oriental Yeast Co, Ltd, Tokyo) and tap water ad libitum and acclimatized for 1 week before the experiment.

The experimental groups and the schedule are shown in Fig 1. Two $\mathrm{mg} / \mathrm{kg}$ body weight CDDP (Randa ${ }^{\circledR}$ Injection; Nippon Kayaku Co, Ltd, Tokyo) was administered intraperitoneally once a week seven times to induce renal injury in the treated rats. Untreated rats in the normal group received intraperitoneal saline.

Oral hydration was performed for 3 days from the CDDP-injection day by three oral administrations at a dose of $20 \mathrm{~mL} / \mathrm{kg}$ for each of the hydration solutions just after administration and one hour before and after CDDP administration. The oral hydration volume of $60 \mathrm{~mL} / \mathrm{kg}$ in this study is extrapolated to a human equivalent dose of 9-10 $\mathrm{mL} / \mathrm{kg}$, considering the difference of body surface area between species. ${ }^{14,15)}$ Rats in the control group received no hydration. We used three hydration solutions: distilled water (Water group), ORS (OS-1 ${ }^{\circledR}$, Otsuka Pharmaceutical Factory, Inc, Naruto, Japan) (ORS group), and modified ORS (MOS group), in which chloride ions were replaced by phosphates. The compositions of ORS and modified ORS are shown in Fig 1. All animals were kept under conditions similar to those of the acclimatization period and their body weights were measured regularly during the ex- 
Treatment Groups

\begin{tabular}{clll}
\hline \multirow{2}{*}{ Group } & \multicolumn{2}{c}{ Treatment } & N \\
\cline { 2 - 3 } & CDDP administration & Oral hydration & \\
\hline \multirow{2}{*}{ Normal } & No (saline injected) & No (free access to water) & 8 \\
Control & CDDP, $2 \mathrm{mg} / \mathrm{kg}$, weekly for 7 weeks & No (free access to water) & 8 \\
Water & CDDP, $2 \mathrm{mg} / \mathrm{kg}$, weekly for 7 weeks & Water, $20 \mathrm{ml} / \mathrm{kg} \times 3$ & 8 \\
ORS & CDDP, $2 \mathrm{mg} / \mathrm{kg}$, weekly for 7 weeks & OS-1, $20 \mathrm{ml} / \mathrm{kg} \times 3$ & 8 \\
MOS & CDDP, $2 \mathrm{mg} / \mathrm{kg}$, weekly for 7 weeks & Modified OS $-1,20 \mathrm{ml} / \mathrm{kg} \times 3$ & 8 \\
\hline
\end{tabular}

Animals

ơF344 rats, $7 \mathrm{Wk}$

CDDP administration

$2 \mathrm{mg} / \mathrm{kg}$ ip, once a week for 7 weeks

Oral hydration

$20 \mathrm{ml} / \mathrm{kg}$ po, 3 times (see below)

Composition of ORS

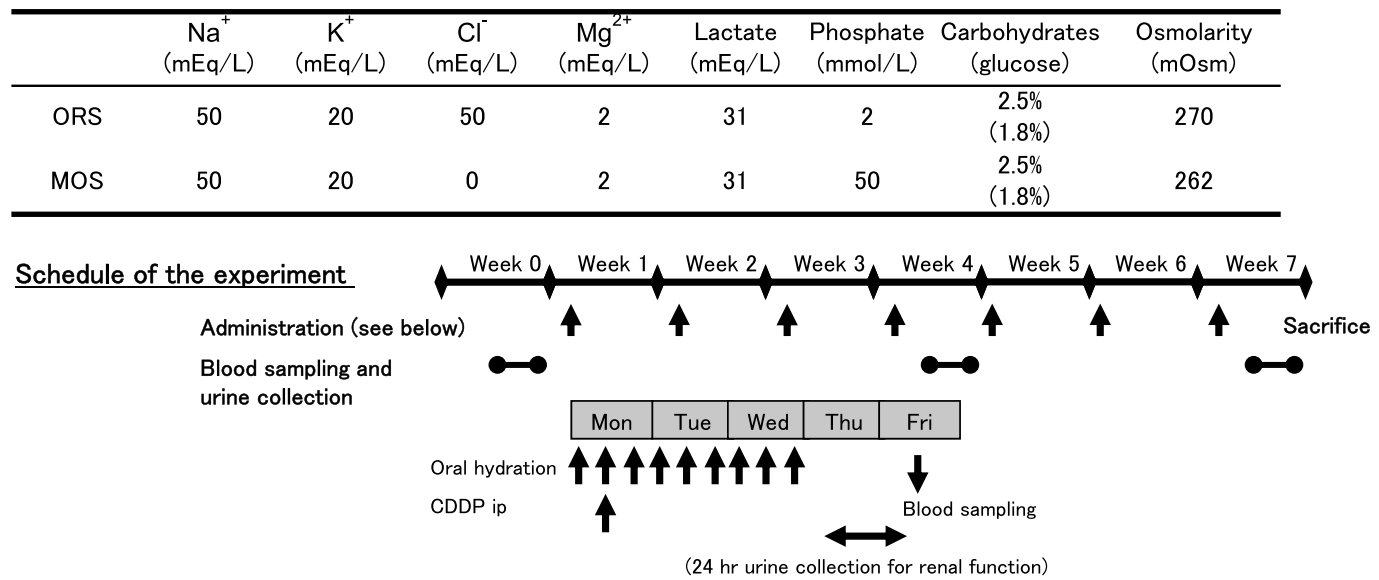

Fig 1 Schedule of the experiment

Fig 1 shows animals, CDDP dosage, oral hydration volume, administration conditions in each treatment groups (Normal, Control, Water, ORS, MOS), composition of ORS and schedule of the experiment.

periment. The experiment received prior approval from the Animal Care and Use Committee at Otsuka Pharmaceutical Factory, Inc.

\section{Evaluation of renal function}

Creatinine in the plasma and creatinine clearance were measured to evaluate renal functions before CDDP administration and at 4 and 7 weeks after the first administration. Urine was collected for 24 hours 3 days after CDDP administration, and blood samples were obtained from the subclavian vein after urine collection. Creatinine in the plasma and the urine was analyzed using an autoanalyzer (7180, Hitachi High-Technologies Corporation, Tokyo). The creatinine clearance was calculated as: urine creatinine $\times$ urine volume/plasma creatinine.

\section{Histopathological evaluation}

At autopsy after final blood sampling at the seventh week, kidney weight was measured and kidney was fixed in 10\% neutral-buffered formalin. Paraffin sections were stained with hematoxylin and eosin. The degree of renal injury was semiquantitatively assessed by an independent pathologist. The semiquantitative assessment was performed as following: “-” if no changes were noticed, “ \pm ” if injuries were found slight (less than $5 \%$ of the observed area), “+” if injuries were found mild (5-20\% of the observed area), " $3+$ " if injuries were found severe (more than $50 \%$ of the observed area), and " $2+$ " for intermediate injury between + and $3+$. 


\section{Urinary platinum excretion}

The effect of oral hydration on urinary cisplatin excretion was studied in a setting similar to that for the renal injury model described above. Fifteen male Fischer-344 rats weighing 220-240 g were intraperitoneally injected with CDDP. Oral hydration was performed using water or ORS by the same schedule as shown in Fig 1. Rats in the control group received no hydration. Urine was collected in two fractions (first 2 hours : fraction 1 and from 2 to 8 hours after CDDP administration : fraction 2), weighed, and subjected to analysis. Urinary platinum was determined by ICP optical emission spectrometry (SPS5520, Hitachi High-Tech Science Corporation, Tokyo), and urinary chloride concentration was determined by the electrode method (EA07, A\&D Co, Ltd, Tokyo).

For urinary platinum analysis, $0.2 \mathrm{~mL}$ of urine sample was mixed with $0.02 \mathrm{~mL}$ of $5 \mathrm{ppm}$ yttrium standard solution as an internal standard element prepared from 1,000 ppm standard stock solution (Kanto Chemical Co, Ltd, Tokyo), subjected to thermolysis after adding sulfuric acid and nitric acid, dissolved in nitric acid, hydrochloric acid, and water to a volume of $10 \mathrm{~mL}$, and then applied for analysis. Standard curve samples were prepared using urine from normal rats mixed with 5 ppm platinum standard solution made from 1,000 ppm standard stock solution (Kanto Chemical Co, Ltd) and yttrium internal standard.

\section{Statistical evaluation}

The measured values are expressed as mean and standard deviation. The significance of differences between the Normal and Control groups was determined using two-tailed Student's $t$-test. Dunnett's procedure for multiple comparisons was used for comparisons of Control vs Water, ORS and MOS, and ORS vs Water and MOS.
P-values less than 0.05 were considered significant. All statistical analyses were performed using SAS version 9.1.3 (SAS Institute Ltd, Tokyo).

\section{Results}

\section{Effect of oral hydration on CDDP-in- duced nephrotoxicity}

Body weight changes of normal and CDDPtreated rats are shown in Fig 2. Body weight of the rats in the Control group was significantly suppressed on day 10 and after day 17 of the first CDDP administration compared with that in the Normal group. Among CDDP-treated groups, body weight suppression was the smallest in the ORS group. Body weight in the ORS group was significantly higher than that in the Water group after day 38 and that in the MOS group after day 42 of the first CDDP administration. One rat in the Water group died on day 45, probably because of deterioration of its general condition due to acute renal failure.

Significant increase of plasma creatinine was found in the Control group compared with that in the Normal group at weeks 4 and $7(P<0.001)$. The reason why the creatinine clearance in the Normal group was increased with time is not clear, but we assume that body weight gain in the Normal group has some relevance with that phenomenon. Plasma creatinine in the ORS group at weeks 4 and 7 was significantly lower than that in the Control group $(P<0.01)$. However, there was no significant decrease of plasma creatinine in the Water and MOS groups compared with the Control group. Plasma creatinine in the ORS group was significantly lower than that in the Water group at both week 4 and week $7(P<0.05)$ (Fig 3A).

Amelioration of renal injury in the ORS group 


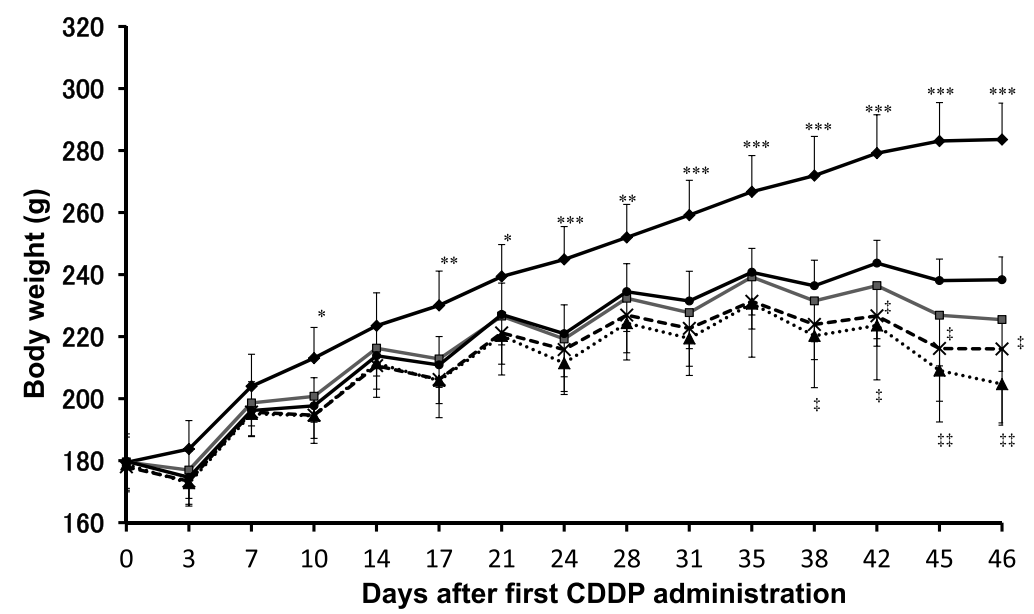

Fig 2 Body weight change during cisplatin administration Fig 2 shows body weight change during cisplatin administration in each treatment groups $\checkmark$ Normal, $\square$ Control, $\boldsymbol{\Delta}$ Water, 0 ORS, $\times$ MOS). Indication of the statistically significant differences were shown by ${ }^{*} P<0.05,{ }^{* *} P<0.01,{ }^{* * *} P<0.001$ (Normal vs Control, Student's $t$-test) and ${ }^{\ddagger} P<0.05,{ }^{\ddagger \ddagger} P<0.01$ (ORS vs Water or MOS, Dunnett).

(A)

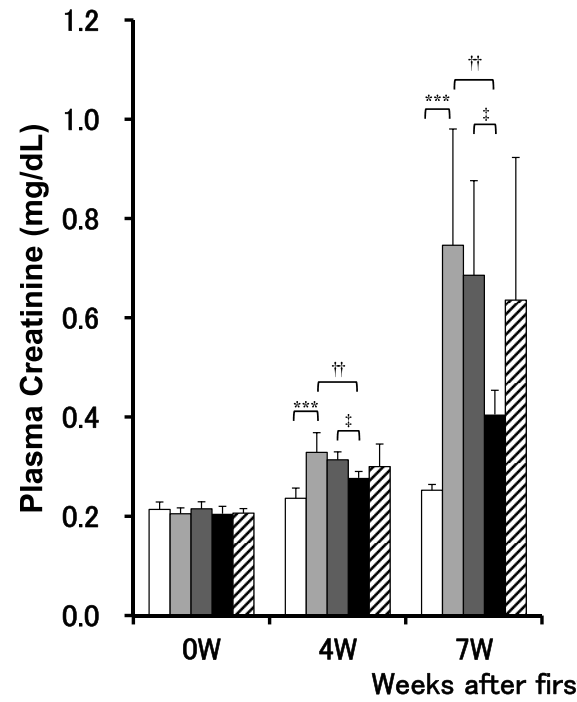

(B)

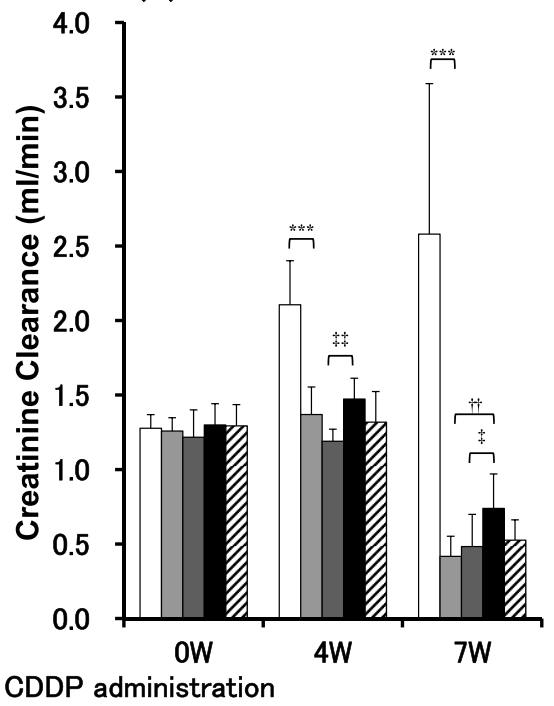

Fig 3 Renal functions during cisplatin administration Fig 3 shows comparisons of plasma creatinine (A) and creatinine clearance (B) in each treatment groups ( $\square$ Normal, $\square$ Control, $\square$ Water, $\square$ ORS, $\square$ MOS). Indication of the statistically significant differences were shown by ${ }^{* * *} P<0.001$ (Normal vs Control, Student's $t$-test), ${ }^{\dagger \dagger} P<0.01$ (Control vs Water, ORS or MOS, Dunnett), ${ }^{*} P<0.01,{ }^{*} P<0.05$ (ORS vs Water or MOS, Dunnett).

was also observed in terms of creatinine clearance. There was a significant decrease of creatinine clearance in the Control group compared with that in the Normal group at weeks 4 and 7 $(P<0.001)$. Creatinine clearance in the ORS group was significantly higher at week 7 than that in the Control group $(P<0.01)$, and was significantly higher than that in the Water group at weeks 4 and 7 ( $P<0.01$ and $P<0.05$, respectively). However, there was no significant difference of creatinine clearance in the Water and MOS groups compared with the Control group (Fig 3B). 
Histopathological examination of the injured kidney revealed tubular necrosis and regeneration in the proximal tubules accompanied by tubular dilation and hyaline cast located in the outer stripe of the outer medulla in the CDDP-treated rats. These findings were graded semiquantitatively using five stages as described in the methods section (Table 1). The degree of tubular injury was the most remarkable in the Control group, followed by the Water group and the MOS group, but the injury in the ORS group was the least severe among the CDDP-treated groups. Typical histological images of the kidney are shown in Fig 4. In the low magnification picture presented in the left panel, the degree of renal injury was determined as - for Fig 4A, 2+ for Fig 4B, + for Fig 4C, \pm for Fig 4D, and $<$ for Fig 4E. The right panel shows pictures with high magnification of the injured area. The tubular degeneration with tubular dilation and hyaline casts were observed in the injured area.

Relative kidney weight at autopsy showed a significant increase in the Control group compared with that in the Normal group $(P<0.001)$, which suggests renal edema (Fig 5). In contrast, relative kidney weight in the ORS group was significantly lower than those in the Control group and the MOS group $(P<0.05)$.

Table 1 Histopathological injury grade of the proximal renal tubule

\begin{tabular}{lccccc}
\hline \hline \multirow{2}{*}{ Group } & \multicolumn{5}{c}{ Histopathological injury grade } \\
\cline { 2 - 6 } & - & \pm & + & $2+$ & $3+$ \\
\hline Normal & 8 & 0 & 0 & 0 & 0 \\
Control & 0 & 0 & 5 & 3 & 0 \\
Water & 0 & 2 & 5 & 0 & 0 \\
ORS & 0 & 8 & 0 & 0 & 0 \\
MOS & 0 & 1 & 5 & 1 & 1 \\
\hline
\end{tabular}

Each column indicates the number of animals with each injury grade. Histopathological injury grade was indicated as -: normal, \pm : slight injury, + : mild injury, $2+$ : intermediate injury, $3+$ : serious injury.
(A)

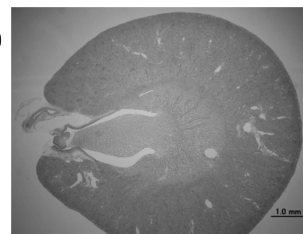

(B)

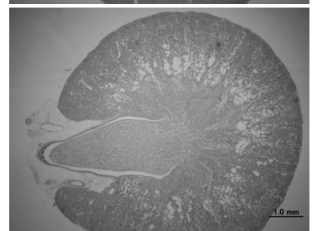

(C)

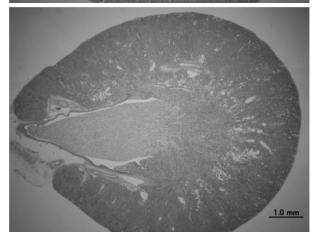

(D)

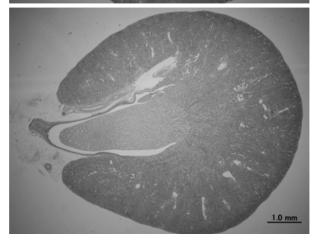

(E)

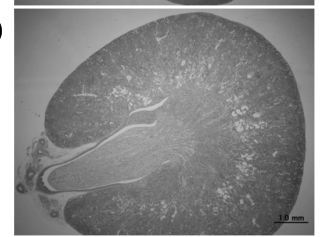

(F)

(G)

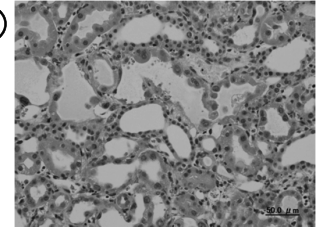

( $\mathrm{H})$

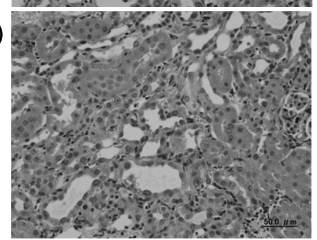

(I)

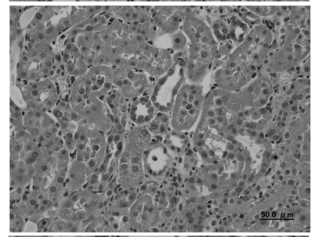

(J)

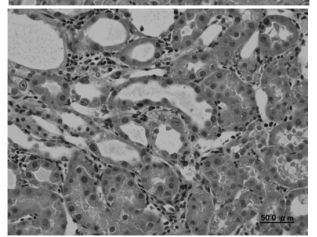

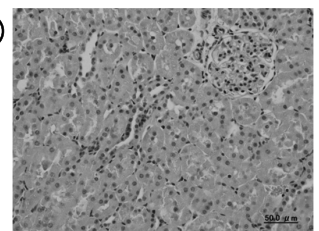

Fig 4 Histopathological examination of the kidney

Fig 4 shows hematoxylin eosin stain of the kidney in each treatment groups (A and F: Normal, B and G: Control, C and H: Water, D and I: ORS, E and J: MOS). Original magnification was $\times 16$ in A, B, C, D, $\mathrm{E}$ and $\times 320$ in $\mathrm{F}, \mathrm{G}, \mathrm{H}, \mathrm{I}, \mathrm{J}$.

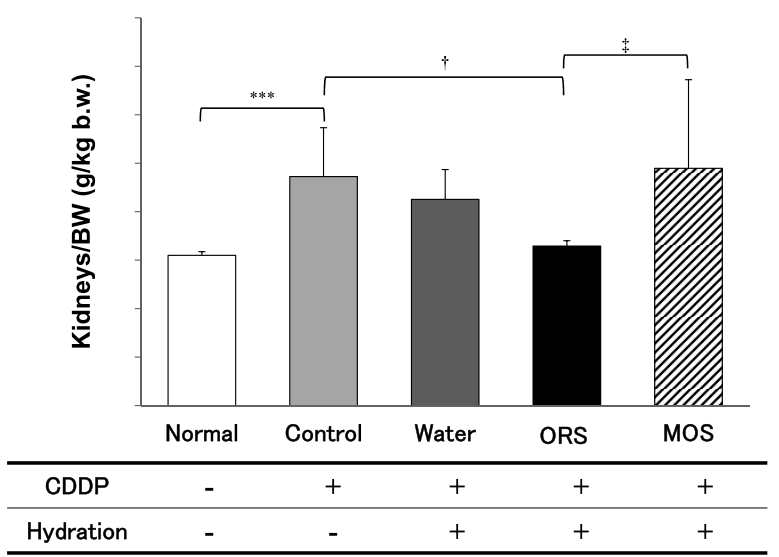

Fig 5 Relative weight of the kidneys

Fig 5 shows comparison of the relative weight of kidneys at the autopsy in each treatment groups ( $\square$ Normal, $\square$ Control, $\square$ Water, $\square$ ORS, $\mathscr{Z} \mathrm{MOS}$ ). Indication of the statistically significant differences were shown by ${ }^{* * *} P<0.001$ (Normal vs Control, Student's $t$-test), ${ }^{\dagger} P$ $<0.05$ (Control vs Water, ORS or MOS, Dunnett), ${ }^{*} P<0.05$ (ORS vs Water or MOS, Dunnett). 


\section{Urinary excretion of cisplatin}

The effect of oral hydration on urinary CDDP excretion was studied by measuring the platinum concentration in the urine after the first CDDP administration (Fig 6). Urine volume was significantly increased in the orally hydrated group compared with that in the Control group in fraction 1 (first 2 hours after CDDP administration) and fraction 2 (2 to 8 hours after CDDP administration), but there was no difference in urine volume between the Water group and the ORS group in both fraction 1 and fraction 2 (Fig 6A). The excretion of platinum in the urine of fraction 1 was significantly greater in the ORS group than in the Control group $(P<0.05)$. In contrast, platinum excretion in fraction 2 was significantly greater in the Control group than in the ORS group $(P<0.05)$. There was no significant difference of total platinum excretion between the Water group and the ORS group (Fig 6B). The urinary chloride concentration in the ORS group was significantly higher than that in the Water group both in fraction $1(P<0.05)$ and in fraction
$2(P<0.05)$. Urinary chloride concentration in the Control group was also significantly higher than that in the Water group in both fraction 1 and fraction $2(P<0.01)$, but this seemed to be a reflection of the urine volume (Fig 6C).

\section{Discussion}

Recently, cancer chemotherapies in outpatient care have been accepted in many hospitals for the purpose of shortening the hospitalization period and improving the quality of life of patients. These trends are also the case for CDDP-containing chemotherapy, but it remains insufficiently widespread in Japan because of a large hydration volume and long-term infusion.

In order to carry out CDDP-containing chemotherapy in an outpatient setting, it is necessary to overcome nausea and vomiting, as well as renal injury, which are dose-limiting factors of CDDP administration. Regimens including carboplatin are adopted in place of CDDP to avoid its demerits, but carboplatin might not be equivalent to
(A)

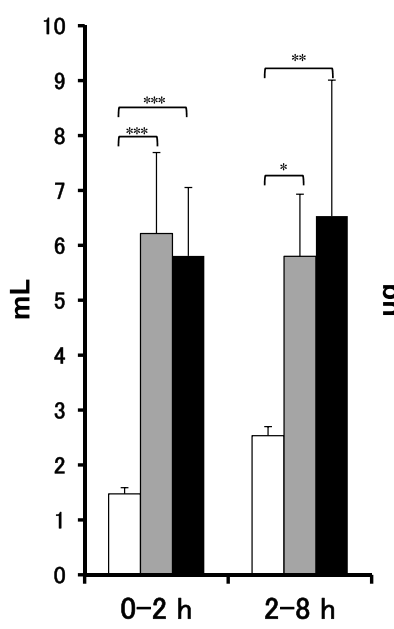

(B)

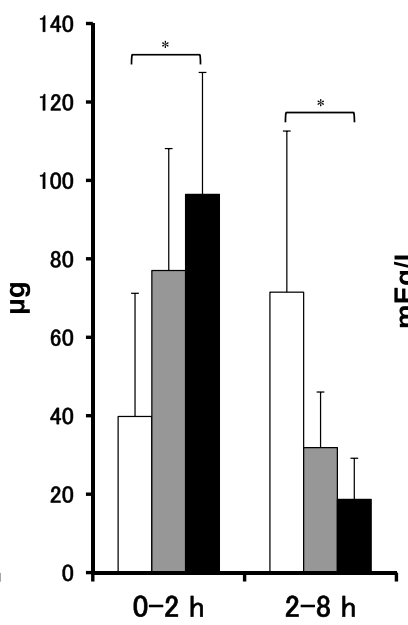

(C)

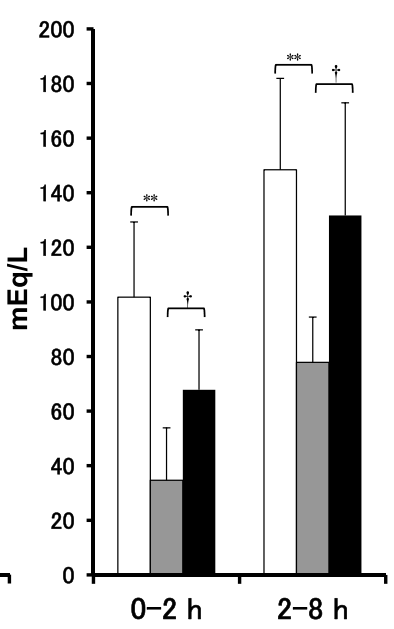

Fig 6 Urine volume (A), platinum excretion (B), and chloride concentration (C) in the urine Fig 6 shows comparisons of urine volume (A), platinum excretion (B), and chloride concentration (C) in each treatment groups ( $\square$ Control, $\square$ Water, $\square$ ORS). Indication of the statistically significant differences were shown by ${ }^{*} P<0.05{ }^{* *} P<0.01{ }^{* * *} P<0.001$ (Dunnett vs Control), ${ }^{\dagger} P<0.05$ (Water vs ORS, Student $t$-test). 
CDDP in terms of its effect. CDDP-based chemotherapy was slightly superior to carboplatin-based chemotherapy in terms of response rate and prolonging survival, without being associated with an increase in severe toxic effects. ${ }^{16}$

The recent introduction of antiemetic drugs such as $5-\mathrm{HT}_{3}$ and NK-1 receptor antagonists has allowed the control of emesis after CDDP administration. Antiemetic guidelines suggest using a combination of 5- $\mathrm{HT}_{3}$ and NK-1 receptor antagonists, as well as dexamethasone to prevent chemotherapy-induced nausea and vomiting in patients receiving highly emetogenic chemotherapy. ${ }^{17}$ However, in terms of CDDP-induced renal injury, the instructions for CDDP indicate 2500-5000 $\mathrm{mL}$ of infusion for 10 hours in total during CDDP-containing regimen to reduce renal toxicity. Compliance with this guideline is a major factor that interferes with the application of CDDP regimens in an outpatient setting in Japan.

Tiseo et al reported that high-dose cisplatin administration at over $75 \mathrm{mg} / \mathrm{m}^{2}$ in lung cancer patients was feasible without a high risk of nephrotoxicity in outpatient management with a short hydration regimen, which included a total infusion volume of $2,100 \mathrm{~mL}$ in four hours $15 \mathrm{~min}$ utes with control of dieresis. ${ }^{2)}$ A short hydration regimen like that in this report is common in most institutions in western countries, and patients are usually treated as outpatients. St Lorant et al suggested that post-hydration could safely be given orally after intravenous pre-hydration to ensure adequate renal blood flow at the time when cisplatin is given. ${ }^{5)}$ Dana et al suggested that the safety of oral hydration is comparable to that of intravenous hydration. ${ }^{4)}$ Oral hydration could be carried out with liquids such as soup, juice, water, coffee, or electrolyte solution, ${ }^{18)}$ but the role and appropriate drink for oral hydration are still unclear and further evaluations are needed. ${ }^{19)}$

The accumulation of CDDP in renal tubular cells and the associated cell death contribute to cisplatin-induced nephrotoxicity. Vigorous hydration in combination with diuresis prevents nephrotoxicity through reducing urinary CDDP concentrations and shortening the retention time within the renal tubules by promoting excretion in an early period after CDDP administration. ${ }^{20)}$ As CDDP-induced renal toxicity is related to the protein-unbound free form of CDDP and this form in plasma is negligible two hours after administration, dilution and excretion of the free form within the early period after CDDP administration are important to prevent renal injury. ${ }^{21}$

In our previous study, oral hydration with ORS but not with water prevented CDDP-induced renal injury in rats, ${ }^{12)}$ suggesting that ORS is an appropriate beverage for oral hydration. This study investigated the mechanism of the protective effect of ORS from the perspectives of urinary CDDP excretion and supplementation of chloride ions via ORS.

The degree of renal injury evaluated by plasma creatinine, creatinine clearance, and histopathological examination in the ORS group was the least severe among CDDP-treated groups, suggesting a protective effect of ORS and confirming the findings in our previous study. In this study, these results were reproduced. Based on the result of a measurement of platinum excretion and chloride ions concentrations in the urine, we discuss the possible mechanisms behind our results below.

First, as the absorption of ORS from the small intestine is faster than that of water, ${ }^{22)}$ faster excretion and dilution in urine of the free form of CDDP might occur after oral hydration with ORS. Although the urine volume was increased in the hydrated group and the excretion of platinum 
in the urine of the early period (within 2 hours after CDDP administration) was significantly higher in the ORS group than in the Control group, there was no significant difference between Water and ORS groups in these parameters, suggesting that the promotion of urinary CDDP excretion might not the mechanism behind the difference between water and ORS.

Second, magnesium included in the ORS might have prevented CDDP-induced renal injury. However, we consider this possibility is unlikely. It was reported that a hydration protocol comprising $8 \mathrm{mEq}$ of magnesium supplementation prevented the nephrotoxicity induced by cisplatin without affecting the treatment outcome. ${ }^{23)}$ As the magnesium concentration of the ORS is only $2 \mathrm{mEq} / \mathrm{L}$ and not all of the administered magnesium is absorbed from the intestine, the amount of supplemented magnesium seemed insufficient to prevent nephrotoxicity.

Third, chloride ion supplementation via ORS might have prevented the formation of the reactive aquated form of CDDP, which is thought to cause tubular cell injury. ${ }^{24)}$ Indeed, CDDP-induced renal injury was prevented in rats that were given chloride salts to induce chloruresis. ${ }^{25)}$ In our experiment, the urinary chloride concentration was higher in the ORS group than in the Water group, which might have contributed to CDDP stabilization and the protective effect of ORS. Conversely, renal injury was not protected against in the chloride-free MOS group. From these results, we speculate that rapid rehydration with chloride supplementation via ORS prevented the reduction in urinary chloride concentration and promoted the stable excretion of CDDP in urine.

The process of concentrating primitive urine also leads to the concentration of CDDP in the tubular fluid, which drives the passive diffusion of
CDDP into tubular cells. ${ }^{26)}$ Hydration is the most effective preventive strategy in the clinical use of CDDP in order to dilute and promote the excretion of CDDP in urine. However, as the use of solutions with a low level of chloride ions, such as plain water, in oral hydration as a substitute for intravenous infusion after CDDP administration may cause activation of CDDP in tubular fluid and then renal injury, care should be taken to supply chloride ions in hydration beverages to promote the stable excretion of CDDP.

The evaluation of the CDDP accumulation in the renal tubular cells is lacking in this study. CDDP enters renal tubular cells through the organic cation transporter-mediated process, leading to disproportionate accumulation of CDDP by processes other than passive diffusion. ${ }^{27)}$ The nephrotoxicity of platinum agents including CDDP was closely associated with their renal accumulation, which is determined by the substrate specificity of the organic cation transporter (OCT) of the basement membrane and multidrug and toxin extrusion (MATE) families of the brush border. ${ }^{28}$ The normal low intracellular chloride concentrations promote formation of the reactive aquated form of CDDP. ${ }^{29)}$ Whether oral hydration with ORS interferes with these processes of the CDDP accumulation and intracellular metabolism in the tubular cells is unknown, thus further studies are needed to clarify the mechanisms involved. In addition, renal blood flow decreases after CDDP administration, probably causing ischemic insult of renal tubular cells. ${ }^{30)}$ Therefore, whether ORS administration improves renal blood flow needs to be clarified. Also, whether chloride ion concentration in the urine affects urinary excretion of CDDP needs to be clarified. These are limitations of this study.

The body weight was decreased in CDDP-treat- 
ed animals. This was due to reduction in food consumption, but not due to dehydration, because the drinking volume in these rats was rather higher than that in normal rats, and the water balance was almost the even among all groups (data not shown).

The levels of decrease in the body weight and the food intake (data not shown) were the smallest in the ORS group among the CDDP-treated groups. This was probably because of amelioration of the general condition through the prevention of renal injury in the rats through the stable excretion of CDDP in the urine after ORS administration. These effects may be expected as benefits of the hydration with ORS. The use of ORS as oral hydration in cancer chemotherapy in an outpatient setting could also contribute to reduce the infusion volume and the duration of hospital stay, as well as to improve the cost, medical resource utilization, and patient's quality of life. ${ }^{31,32)}$ Future clinical evaluations are needed to clarify its safety and usefulness in clinical practice.

In conclusion, our results showed that oral hydration using ORS was more effective than plain water to prevent CDDP-induced renal injury in rats. The mechanisms involved could be the supplementation of chloride ions to promote the stable excretion of CDDP in urine. ORS could be a useful tool to promote CDDP-containing regimens in outpatient cancer chemotherapy.

\section{Acknowledgements}

The correspondence author was involved in this study from a design, a plan to results analysis, article writing directly. For experimental accomplishment, the author received technical support of Ohtsuka Pharmaceutical Factory, Inc which was the affiliation of the joint author. We thank Dr Makoto Tanaka (Naruto Research Institute,
Otsuka Pharmaceutical Factory, Inc) for pathological evaluation and advice.

\section{References}

1) Johnson SW, O'Dwyer PJ, "Pharmacology of cancer chemotherapy: cisplatin and its analogues. In: Cancer. Principles and practice of oncology" 7th ed, De Vita VT Jr, Hellman S, Rosenberg SA (Eds), Lippincott Williams \& Wilkins, Philadelphia, 2005, pp344-358.

2) Tiseo M, Martelli O, Mancuso A, Sormani MP, Bruzzi P, Di Salvia R, De Marinis F, Ardizzoni A, Short hydration regimen and nephrotoxicity of intermediate to high-dose cisplatin-based chemotherapy for outpatient treatment in lung cancer and mesothelioma, Tumori, 2007, 93, 138-144.

3) Brock J, Alberts DS, Safe, rapid administration of cisplatin in the outpatient clinic, Cancer Treat Rep, 1986, 70, 1409-1414.

4) Dana R, Kachhwaha VS, Comparison of oral and intravenous hydration and diuretic, choice for protecting cisplatin induced nephrotoxicity, Indian J Cancer, 1996, 33, 168-170.

5) Saint-Lorant G, Madelaine J, Galais MP, Thierry L, Chédru-Legros V, Hydration of patients treated with cisplatin: practical investigation and establishment of a protocol, Therapie, 2005, 60, 499-505.

6) Yamada K, Yoshida T, Zaizen Y, Okayama Y, Naito Y, Yamashita F, Takeoka H, Mizoguchi Y, Yamada K, Azuma K, Clinical practice in management of hydration for lung cancer patients receiving cisplatinbased chemotherapy in Japan: a questionnaire survey, Jpn J Clin Oncol, 2011, 41, 1308-1311.

7) King CK, Glass R, Bresee JS, Duggan C, Managing acute gastroenteritis among children: oral rehydration, maintenance, and nutritional therapy, MMWR Recomm Rep, 2003, 52, 1-16.

8) Atia AN, Buchman AL, Oral rehydration solutions in non-cholera diarrhea: a review, Am J Gastroenterol, 2009, 104, 2596-2604.

9) Hartling L, Bellemare S, Wiebe N, Russell K, Klassen TP, Craig W, Oral versus intravenous rehydration for treating dehydration due to gastroenteritis in children, Cochrane Database Syst Rev, 2006, 3, CD004390.

10) Goseki N, Hiranuma S, Yamazaki S, Maruyama M, Nakajima K, Gen T, Shirataka M, Oral rehydration solution for providing water and electrolytes following laparoscopic cholecystectomy and recovery of 
intestinal function, Hepatogastroenterology, 2007, 54, 2276-2281.

11) Taniguchi H, Sasaki T, Fujita H, Oral rehydration therapy for preoperative fluid and electrolyte management, Int J Med Sci, 2011, 8, 501-509.

12) Sato J, Kudo K, Hino K, Takahashi K, Usefulness of oral rehydration solution for hydration in cancer chemotherapy: toward the application of outpatient chemotherapy with high-dose Cisplatin therapy-, Gan To Kagaku Ryoho, 2011, 38, 1471-1476.

13) Yao X, Panichpisal K, Kurtzman N, Nugent K, Cisplatin nephrotoxicity: a review, Am J Med Sci, 2007, 334, 115-124.

14) Pinkel $D$, The use of body surface area as a criterion of drug dosage in cancer chemotherapy, Cancer Res, 1958, 18, 853-856

15) Reagan-Shaw S, Nihal M, Ahmad N, Dose translation from animal to human studies revisited, FASEB $J, 2008,22,659-661$.

16) Ardizzoni A, Boni L, Tiseo M, Fossella FV, Schiller JH, Paesmans M, Radosavljevic D, Paccagnella A, Zatloukal P, Mazzanti P, Bisset D, Rosell R, Cisplatin- versus carboplatin-based chemotherapy in firstline treatment of advanced non-small-cell lung cancer: an individual patient data meta-analysis, $J$ Natl Cancer Inst, 2007, 99, 847-857.

17) Basch E, Prestrud AA, Hesketh PJ, Kris MG, Feyer PC, Somerfield MR, Chesney M, Clark-Snow RA, Flaherty AM, Freundlich B, Morrow G, Rao KV, Schwartz RN, Lyman GH, Antiemetics: American Society of Clinical Oncology clinical practice guideline update, J Clin Oncol, 2011, 29, 4189-4198.

18) Frogge $\mathrm{MH}$, Streamlining outpatient cisplatin therapy to meet the challenges of today, Semin Oncol Nurs, 1989, 5, 21-28.

19) Greystoke AP, Jodrell DI, Cheung M, Rivans I, Mackean MJ, How many cisplatin administration protocols does your department use?, Eur J Cancer Care, 2010, 19, 80-90.

20) Daugaard G, Abildgaard U, Cisplatin nephrotoxicity. A review, Cancer Chemother Pharmacol, 1989, 25, $1-9$.

21) Sasaki Y, Tamura T, Eguchi K, Shinkai T, Fujiwara Y, Fukuda M, Ohe Y, Bungo M, Horichi N, Niimi S, Pharmacokinetics of (glycolate-0,0')-diammine platinum (II), a new platinum derivative, in comparison with cisplatin and carboplatin, Cancer Chemother Pharmacol, 1989, 23, 243-246.
22) Nishinaka D, Kishino F, Matsuura A, Water and electrolyte absorption from hypotonic oral rehydration solution in rat small intestine and colon, Pediatr Int, 2004, 46, 315-321.

23) Muraki K, Koyama R, Honma Y, Yagishita S, Shukuya T, Ohashi R, Takahashi F, Kido K, Iwakami S, Sasaki $\mathrm{S}$, Iwase A, Takahashi K, Hydration with magnesium and mannitol without furosemide prevents the nephrotoxicity induced by cisplatin and pemetrexed in patients with advanced non-small cell lung cancer, J Thorac Dis, 2012, 4, 562-568.

24) Daley-Yates PT, McBrien DC, A study of the protective effect of chloride salts on cisplatin nephrotoxicity, Biochem Pharmacol, 1985, 34, 2363-2369.

25) Earhart RH, Martin PA, Tutsch KD, Erturk E, Wheeler RH, Bull FE, Improvement in the therapeutic index of cisplatin (NSC 119875) by pharmacologically induced chloruresis in the rat, Cancer Res, 1983, 43, 1187-1194.

26) Gately DP, Howell SB, Cellular accumulation of the anticancer agent cisplatin: a review, Br J Cancer, 1993, 67, 1171-1176.

27) Yokoo K, Murakami R, Matsuzaki T, Yoshitome K, Hamada A, Saito H, Enhanced renal accumulation of cisplatin via renal organic cation transporter deteriorates acute kidney injury in hypomagnesemic rats, Clin Exp Nephrol, 2009, 13, 578-584.

28) Yokoo S, Yonezawa A, Masuda S, Fukatsu A, Katsura $\mathrm{T}$, Inui $\mathrm{K}$, Differential contribution of organic cation transporters, OCT2 and MATE1, in platinum agentinduced nephrotoxicity, Biochem Pharmacol, 2007, 74, 477-487.

29) Greene RF, Chatterji DC, Hiranaka PK, Gallelli JF, Stability of cisplatin in aqueous solution, Am J Hosp Pharm, 1979, 36, 38-43.

30) Winston JA, Safirstein R, Reduced renal blood flow in early cisplatin-induced acute renal failure in the rat, Am J Physiol, 1985, 249, 490-496.

31) Mor V, Stalker MZ, Gralla R, Scher HI, Cimma C, Park D, Flaherty AM, Kiss M, Nelson P, Laliberte L, Day hospital as an alternative to inpatient care for cancer patients: a random assignment trial, J Clin Epidemiol, 1988, 41, 771-785.

32) Cox KM, Goel S, O'Connell RL, Boyer M, Beale PJ, Simes RJ, Stockler MR, Randomized cross-over trial comparing inpatient and outpatient administration of high-dose cisplatin, Intern Med J, 2011, 41, 172-178. 